\title{
Chapter 4 \\ Determining Ischaemic Thresholds Through Our Understanding of Cellular Metabolism
}

\author{
Alan J. Johnstone and Derek Ball
}

\section{Background to the Chapter}

- Synopsis of clinical issues associated with the complexity of compartment syndromes and current diagnostic limitations

- Comprehending the metabolic capacity of active skeletal muscle and its constant need for energy

- Understanding the principle of an ischaemic threshold and the localised techniques that are employed by muscle to counteract progressive ischaemia

- Investigating the potential for tissue concentrations of key biochemical molecules involved in aerobic and anaerobic respiration to become markers of ischaemia

- Investigating the role of direct tissue $\mathrm{pH}$ monitoring to become a future objective measure of muscle metabolic status related to ischaemia irrespective of the cause

\section{Clinical Issues and Concerns}

Compartment syndromes, through their definition, are complex, multifactorial and ultimately result in irreversible cell damage leading to cell death. Although their causation differs, influenced significantly by a number of local or systemic factors,

\footnotetext{
A. J. Johnstone ( $\square)$

Aberdeen Royal Infirmary \& University of Aberdeen, Orthopedic Trauma Unit, Aberdeen, Grampian Region, UK

e-mail: alan.johnstone@ abdn.ac.uk

D. Ball

School of Medicine, University of Aberdeen, Medical Sciences and Nutrition, Aberdeen, Aberdeenshire, UK

e-mail: derek.ball@abdn.ac.uk 
they all have one thing in common - progressive tissue ischaemia that if unchecked results in death of the cells within the affected limb [1]. The most widely studied and understood form of compartment syndrome is trauma-related compartment syndrome, often referred to as acute compartment syndrome (ACS) and can occur following fractures, soft tissue crush injuries or burns. However, clinicians should also be aware of the significant effects that systemic hypotension or hypoxia can have upon an injured limb that is already compromised, has a higher metabolic need compared with uninjured tissues and is therefore prone to develop this otherwise insidious complication.

With particular reference to ACS, accurately diagnosing this syndrome remains a challenge since the clinical symptoms and signs that accompany early-stage ACS are difficult to differentiate from those that accompany the original injury, and for this reason, there has been considerable interest in developing objective tests that could aid diagnosis and permit earlier intervention resulting in better long-term clinical outcomes. The most commonly used objective method for diagnosing ACS is to measure intracompartmental pressure (ICP) [2] since trauma results in localised swelling that in turn gives rise to an increase in the ICP, which undoubtedly contributes to the underlying soft tissue ischaemia. However, despite the wide acceptance of Matsen's arteriovenous gradient theory behind the pathophysiology of ACS, which elegantly explains the mechanical aspects of this syndrome, it lacks useful information about the underlying cellular effects especially in the presence of injury and the resultant increase in requirements for energy by the injured cells. Overall, the majority of clinicians remain unconvinced about the value of monitoring ICP given its well-documented poor diagnostic specificity, and the search continues for better objective diagnostic methods [3].

\section{Skeletal Muscle Physiology}

Skeletal muscle is a highly metabolically active tissue. Even at rest, it has been calculated that the turnover of adenosine triphosphate (ATP) is around $35 \mu \mathrm{mol} . \mathrm{kg}^{-1}$ muscle, with the energy used mainly to transport $\mathrm{Ca}^{2+}$ and to maintain the balance of intracellular and extracellular $\mathrm{Na}^{+}$and $\mathrm{K}^{+}$across the sarcolemma [4]. However, skeletal muscle can rapidly increase its consumption of ATP by approximately 1000 -fold $\left(5 \mathrm{mmol} . \mathrm{kg}^{-1}\right)$ during maximum exercise with $70 \%$ of the ATP being utilised to undertake muscular work through the interaction of myosin and actin and the remaining $30 \%$ employed to transport $\mathrm{Ca}^{2+}, \mathrm{Na}^{+}$and $\mathrm{K}^{+}[5]$. There is a very limited store of ATP resulting in a constant requirement for ATP to be replenished. When oxygen delivery and availability is abundant, ATP is primarily re-synthesised through oxidative phosphorylation of fatty acids via mitochondrial respiration, but during periods of increased energy turnover, glycogen and glucose are also used as substrates to produce ATP. This process is also known as aerobic respiration. However, when oxygen availability and/or delivery is below that required for oxidative phosphorylation, the energy required to re-synthesise ATP is produced through 
glycolysis (also known as anaerobic respiration); under these circumstances, the end product of glycolysis, pyruvate, is converted to lactic acid, resulting in an accumulation of $\mathrm{H}^{+}$ions intra- and extracellularly. After high-intensity exercise, the metabolic consequence is that intramuscular pH levels can be as low as 6.5 [6]. Although in the liver, lactate can be oxidised to pyruvate and ultimately converted into glucose-6-phosphate which can then be utilised for oxidative phosphorylation. Since the enzymes required for this conversion are not present in the skeletal muscle, lactic acid could be considered a metabolically 'dead end' molecule in muscle and only becomes a useful source of energy when it is transported to the liver where it is reprocessed through gluconeogenesis [7]. To exacerbate matters, given that ACS results in a gradual reduction of venous blood flow, the mechanism for reprocessing of lactate by the liver becomes increasingly restricted resulting in an accelerated build-up of lactic acid within muscle. The $\mathrm{H}^{+}$ions that accumulate intracellularly, as a result of anaerobic respiration, are buffered to some degree by intracellular proteins, although a sizeable proportion are actively transported extracellularly where they can be buffered by plasma bicarbonate and the lactic acid used in the liver as a substrate through gluconeogenesis.

In extreme circumstances when the muscles' need for energy is exceptionally high and glycolysis is insufficient, the high-energy phosphate-containing molecules, phosphocreatine (PCr) and adenosine diphosphate (ADP), can be catabolised [8]. However, this process results in a reduction in the total adenine nucleotide pool [9], a situation that is still reversible but requires considerable future energy reserves to rectify and resembles a pre-terminal stage for the cell.

\section{When Reversible Cell Injury Becomes Irreversible}

Cells are remarkably tolerant to ischaemia, but in the absence of sufficient energy reserves, cell membrane ion exchange pumps become less efficient resulting in an accumulation of $\mathrm{Na}^{+}$intracellularly and a diffusion of $\mathrm{K}^{+}$out of the cells features that are associated with cellular swelling; overall protein synthesis slows; and in muscle there is reduced contractility. However, if the delivery of adequately oxygenated blood is restored, all of these cellular disturbances are reversible.

Irreversible injury is associated with morphological features such as severe swelling of mitochondria, extensive damage to plasma membranes and swelling of lysosomes. These features result in mitochondria that are unable to synthesise ATP and plasma and organelle membrane damage that leads to structural loss of the cell and the organelles resulting in the undesirable entry of extracellular proteins and loss of intracellular proteins. It is at this stage that myocyte specific proteins such as troponin and creatine kinase are released into the extracellular fluid and are useful blood biomarkers of cellular damage. Loss of membrane integrity also results in extracellular $\mathrm{Ca}^{2+}$ entering the cell and in particular the mitochondria. In circumstances where the irreversibly damaged cells are reperfused, $\mathrm{Ca}^{2+}$ is taken up rapidly by the mitochondria and permanently poisons them through inhibiting enzyme 
activity. Also, oxygen free radicals are produced on restoration of the blood supply resulting in further direct injury to the plasma and organelle membranes.

In summary, membrane injury and subsequent significant dysfunction is the central factor leading to irreversible cell injury.

\section{Coping Mechanisms That Are Employed by Skeletal Muscle in Response to Ischaemia}

Hypo-perfusion resulting in localised ischaemia is a fact of everyday life, where cells, tissues and organs are perpetually utilising a variety of coping mechanisms to promote blood flow, varying oxygen extraction from the blood, and to modify cellular metabolism to generate energy depending upon the concentration of oxygen that is locally available.

1. Autoregulation is the term given to the ability of the microcirculation to reduce vascular resistance through relaxing the smooth muscle present within the vessel walls that in turn improves blood flow in situations when the arteriovenous pressure gradient is subnormal. However, this inherent compensatory mechanism is soon overcome by a developing ACS whereby venous blood flow is inhibited by the raised intramuscular pressure, thus lowering the arteriovenous gradient.

2. Another compensatory coping strategy utilised by ischaemic muscle is to extract more oxygen than is the norm from venous blood. In non-ischaemic situations, oxygen delivery is excessive; and therefore, the venous blood contains surplus oxygen that can be utilised when the body is exercising and the demand for oxygen is higher. A decline in $\mathrm{pH}$ will favour an increase in oxygen offloading due to a shift in the haemoglobin dissociation curve, and in the presence of a developing ACS, this mechanism for obtaining additional oxygen is maximised.

3. The third and most important coping mechanism is the ability of all human cells to generate energy in the presence of ischaemia by activating the glycolytic pathway. Although the mechanism is effective, it is not an efficient use of glucose since it results in 12-fold lower production of ATP compared with oxidative phosphorylation and produces lactic acid as a by-product. Glycolysis is employed by all cells on a routine basis and is activated when required to make up any energy shortfall. In the presence of a developing ACS, the glycolytic pathway becomes increasingly important for cell survival, although, through its inefficiencies, it is not sustainable, and ATP production declines to a level where adequate plasma and organelle membrane function is lost, and reversible cell injury becomes irreversible.

\section{Could biochemical markers be indicative of impending irreversible cell injury?}

In principle, if the tissue concentrations of key biochemical markers could be measured accurately, it seems likely that a relationship between their concentration and 
the extent of tissue ischaemia could be established. However, these potential relationships have not been investigated in depth, and to date no studies have been undertaken that directly compare the biochemical composition of muscle with the morphological features of reversible and irreversible cell injury.

Through research undertaken in our unit, we have investigated the potential relationship between progressive ischaemia in skeletal muscle with the tissue concentration of key biomarker molecules (glucose-6-phosphate, pyruvate and lactate) that play central roles in oxidative phosphorylation and glycolysis, and the end products, ATP. The model that we used was a non-circulatory model utilising fresh avascular blocks of mammal skeletal muscle. Although this model is not directly comparable with ACS models, where the blood flow is gradually deteriorating, our model was useful due to its simplicity, consistency and ability to foreshorten the overall experimental time from well-vascularised muscle to irreversible cell injury (death). The experimental model also facilitated the process of obtaining biopsy specimens at regular intervals. After freezing all biopsy specimens in liquid nitrogen and further processing of the specimens, we were able to measure the tissue concentration of each of the aforementioned key molecules and compare them in relation to ischaemic duration and level of tissue $\mathrm{pH}$ with the latter being calculated using the aerobic-anaerobic equation described by Sahlin [10] and used to determine the extent of ischaemia. The aerobic-anaerobic equation is dependent upon the concentrations of lactate and pyruvate, which are key intermediate and end molecules in the glycolytic and oxidative pathways. They are therefore indicative of the balance between anaerobic and aerobic respiration. Tissue concentrations of ATP and PCr were used to determine when the cell energy reserves were depleted to the point of irreversible cell injury and cell death was imminent.

In summary, over time, glucose-6-phosphate, pyruvate levels and $\mathrm{PCr}$ decline predictably as these key energy substrates are used to make ATP (Figs. 4.1, 4.2, and 4.3). In response to the glycolytic activity, lactate levels increase over time (Fig. 4.4). Finally, despite the concerted effort to maintain ATP re-synthesis, the metabolic consequence of ischaemia still results in a $\sim 75 \%$ decline ATP concentration after 90 minutes (Fig. 4.5).

In addition, when the tissue concentrations of all of these molecules are plotted against tissue $\mathrm{pH}$ (data not shown), there is a strong correlation (and a reverse correlation for lactate) between both $\mathrm{pH}$ and the concentrations of the aforementioned key molecules.

\section{Assuming that there is a relationship between the concentration of key biochemical markers and the extent of muscle ischaemia, could any of these markers be used as an objective measure of ischaemia?}

Our research strongly suggests that a number of these biochemical markers could be useful in determining the extent of tissue ischaemia, irrespective of the cause, but the difficulty lies in how best to measure their concentration in tissues in the clinical setting. Microdialysis is one method that could be employed, but this technique which can be used to measure the extracellular concentration of molecules of interest would be questionable under conditions when an increase in the intramuscular pressure may 


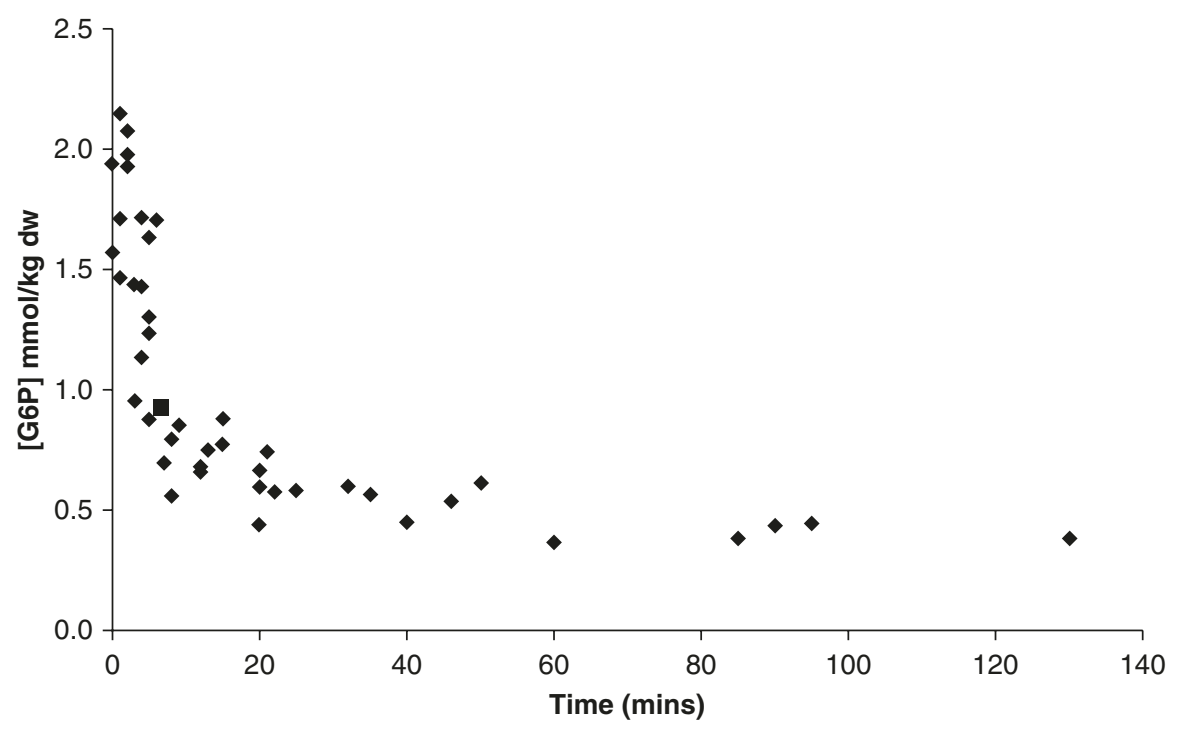

Fig. 4.1 Ischaemic mammalian animal muscle study: glucose-6-phosphate concentration against time

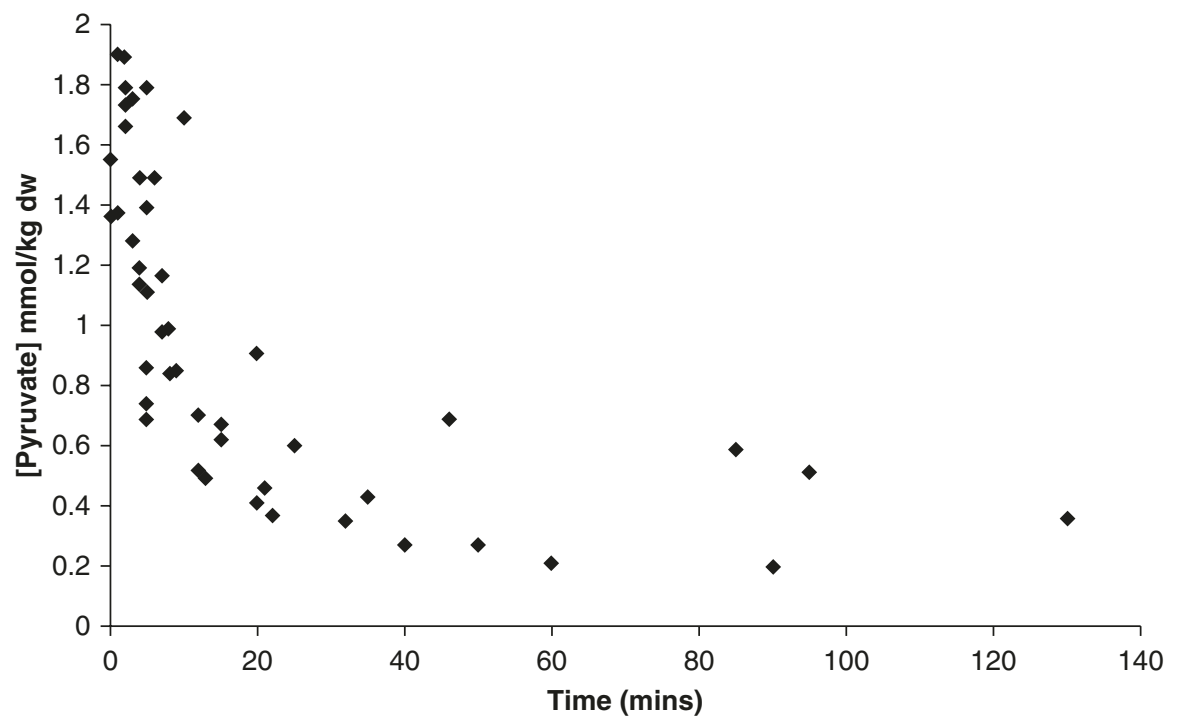

Fig. 4.2 Ischaemic mammalian animal muscle study: pyruvate concentration against time

influence the movement of biomolecular markers in contrast to normal conditions. In principle, spectrophotometric methods for determining the tissue concentration of these key molecules hold promise but would require multi-wavelength optical analysis for each metabolite of interest and as such are not sufficiently well advanced to be 


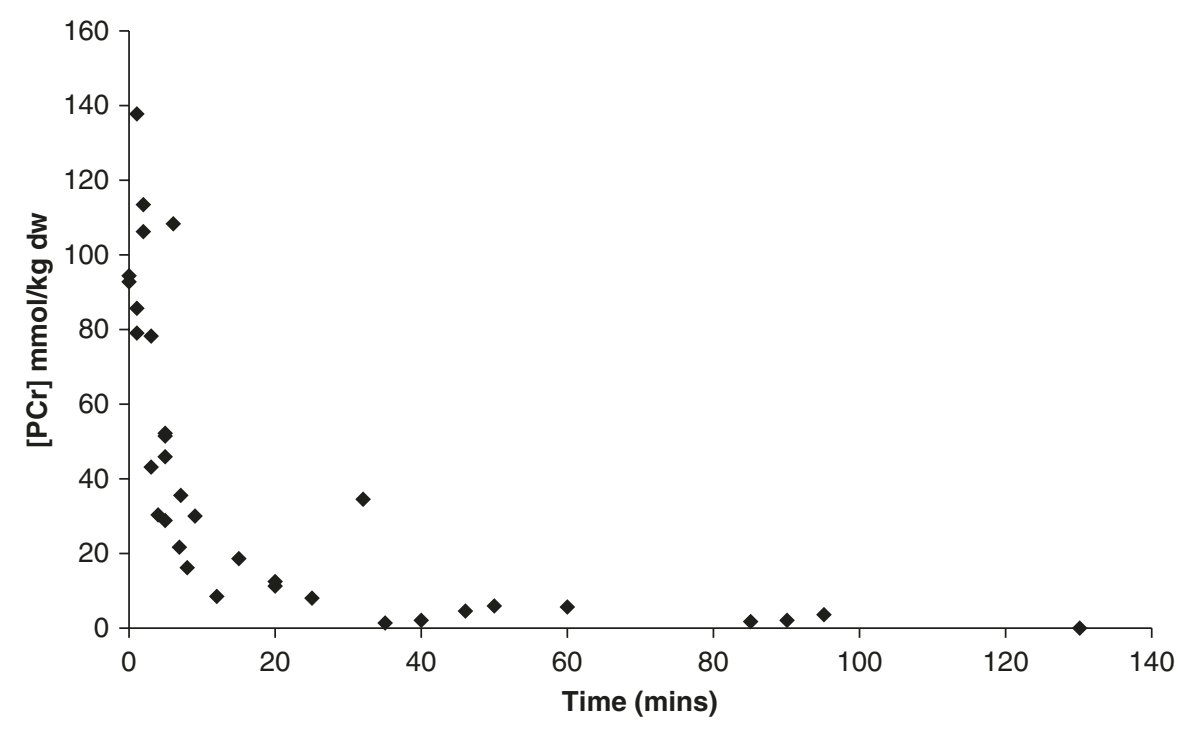

Fig. 4.3 Ischaemic mammalian animal muscle study: phosphocreatine concentration against time

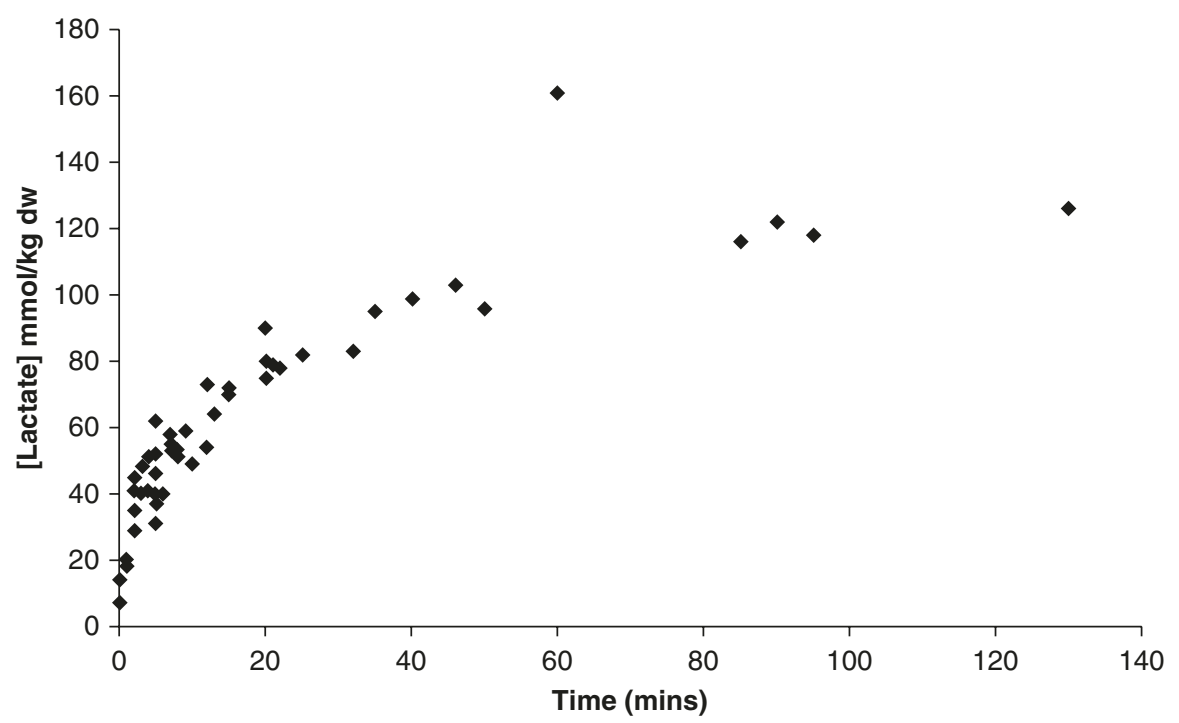

Fig. 4.4 Ischaemic mammalian animal muscle study: lactate concentration against time

of use. Even the more established spectrophotometric technique for determining the oxygen concentration in tissues, near-infrared spectroscopy (NIRS) is not proving to be as useful as was first anticipated due to a number of confounding factors.

One method that we have been investigating in more detail is the potential of measuring $\mathrm{pH}$ directly in muscle. Tissue acidity $(\mathrm{pH})$ is a direct reflection of the 


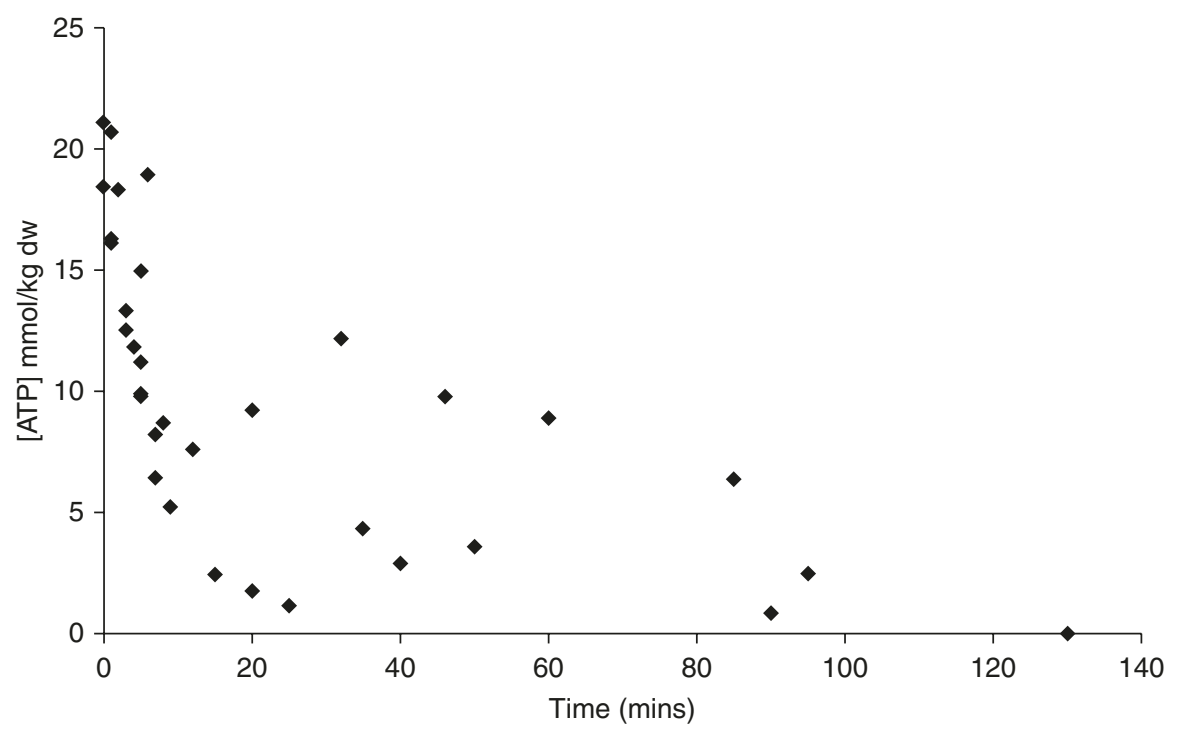

Fig. 4.5 Ischaemic mammalian animal muscle study: ATP concentration against time

concentration of $\mathrm{H}^{+}$ions present, and although the $\mathrm{H}^{+}$ion is not one of the key molecules, it does represent a reasonably accurate measure of glycolysis that has been taking place. In addition to the aforementioned biochemical analyses, our experiments also assessed the accuracy of measuring directly the tissue $\mathrm{pH}$ of muscle using $\mathrm{pH}$ probes. Our results confirmed a strong correlation $\left(R^{2}=0.926\right)$ between directly measured $\mathrm{pH}$ with calculated $\mathrm{pH}$ based upon the tissue concentration of lactate and pyruvate, using the aerobic-anaerobic equation. Although more work is required to confirm the earlier findings, it is possible that directly measured tissue acidity using a $\mathrm{pH}$ probe may prove to be a useful objective measure of muscular metabolic status related to ischaemia that could have roles in diagnosing and assessing the severity of ACS in addition to other forms of compartment syndrome [11].

\section{Limitations of Current Knowledge and Future Directions}

- It is clear that further research needs to be done in an attempt to directly associate the concentrations of key cellular metabolic markers with the morphological changes that are recognised for reversible and irreversible cell injury. If key metabolic biomarkers can be identified, these will open the door to the development of new objective measures of ischaemia.

- Based on our research (laboratory and clinical), monitoring intramuscular pH seems to be a highly accurate method (high sensitivity and specificity) for detecting muscle ischaemia and the metabolic status of the muscle and appears to have 
advantages over ICP monitoring. A future RCT will be required to assess the potential of IMpH monitoring to diagnose ACS and other forms of compartment syndrome [12].

\section{Take-Home Message}

- Skeletal muscle is a highly metabolically active tissue that has a constant demand for energy.

- Underlying cellular metabolic pathways are well established for both aerobic and anaerobic environments.

- Morphological changes in cellular structure have also been well described for both reversible and irreversible cell damage secondary to ischaemia.

- Identifying key stages in the cellular metabolic response to ischaemia that can differentiate between reversible and irreversible cell damage (ischaemic threshold) should be possible.

- Identifying an ischaemic threshold is of fundamental importance if new diagnostic methods are to be developed that would aid the decision-making process of clinicians responsible for treating patients with a suspected compartment syndrome or other forms of peripheral ischaemia.

- Early research suggests that direct intramuscular $\mathrm{pH}$ monitoring could be a suitable objective measure of muscle ischaemia including compartment syndrome.

- Despite enthusiastic attempts to diagnose peripheral ischaemia by measuring key biochemical markers in venous or arterial blood, dilutional effects significantly dampen the responsiveness of potential markers except in extreme situations where extensive ischaemic damage resulting in cell death is present, by which time, treatment is aimed at tissue/limb salvage at best.

\section{References}

1. Elliott KGB, Johnstone AJ. Diagnosing acute compartment syndrome. Bone Joint J. 2003;85-B:625-32.

2. Williams PR, Russell D, Mintowt-Cryz WJ. Compartment pressure monitoring - current UK orthopaedic practice. Injury. 1998;29:229-32.

3. Wall CJ, Richardson MD, Lowe AJ, Brand C, Lynch J, de Steiger RN. Survey of management of acute traumatic compartment syndrome of the leg in Australia. ANZ J Surg. 2007;9:733-7.

4. Smith IC, Bombardier E, Vigna C, Tupling AR. ATP consumption by sarcoplasmic reticulum $\mathrm{Ca} 2+$ pumps accounts for $40-50 \%$ of resting metabolic rate in mouse fast and slow twitch skeletal muscle. PlosOne. 2013;8(7):e68924. PMID: 23840903.

5. Hultman E, Spriet LL. Skeletal muscle metabolism, contraction force and glycogen utilization during prolonged electrical stimulation in humans. J Physiol. 1986;374:493-501. Hultman E, Sjoholm H. Energy metabolism and contraction force of human skeletal muscle in situ during electrical stimulation. J Physiol. 1983;345:525-32. 
6. Jones N. Hydrogen ion balance during exercise. Clin Sci. 1980;59:85-91.

7. Wahren J, Felig P, Ahlborg G, Jorfeldt L. Glucose metabolism during exercise in man. J Clin Invest. 1972;50:2715-25.

8. Ball D. Metabolic and endocrine response to exercise: sympathoadrenal integration with skeletal muscle. J Endocrinol. 2015;224:R79-95; Meyer RA, Terjung RL. AMP deamination and IMP reamination in working skeletal muscle. Am J Phys. 1980;239:C32-8.

9. Patton MS. A review of the underlying biochemistry of muscle ischaemia in relation to intramuscular $\mathrm{pH}$ measurements. MD Thesis, University of Aberdeen, 2008. (unpublished).

10. Sahlin K. Muscle energetics during explosive activities and potential effects of nutrition and training. Sports Med. 2014;44:S167-73.

11. Elliott KGB. Intramuscular $\mathrm{pH}$ as a novel diagnostic tool for acute compartment syndrome: a prospective clinical study. MD Thesis, University of Aberdeen, 2007.

12. Schmidt AH, Bosse MJ, Frey KP, O’Toole RV, Stinner DJ, Scharfstein DO, Zipunnikov V, MacKenzie EJ, METRC. Predicting acute compartment syndrome (PACS): the role of continuous monitoring. J Orthop Trauma. 2017;31(Suppl 1):S40-7.

Open Access This chapter is licensed under the terms of the Creative Commons Attribution 4.0 International License (http://creativecommons.org/licenses/by/4.0/), which permits use, sharing, adaptation, distribution and reproduction in any medium or format, as long as you give appropriate credit to the original author(s) and the source, provide a link to the Creative Commons license and indicate if changes were made.

The images or other third party material in this chapter are included in the chapter's Creative Commons license, unless indicated otherwise in a credit line to the material. If material is not included in the chapter's Creative Commons license and your intended use is not permitted by statutory regulation or exceeds the permitted use, you will need to obtain permission directly from the copyright holder.

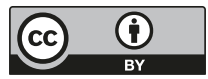

